\title{
The lattice and rotational dynamics of the methyl halides described by pair potentials based on universal force fields
}

\author{
O. Kirstein ${ }^{\mathrm{a})}$ \\ Bragg Insitute, Australian Nuclear Science \& Technology Organisation, Menai, NSW 2243, Australia, \\ and Institut für Festkörperforschung, Forschungszentrum Jülich GmbH, D-52425 Jülich, Germany \\ M. Prager \\ Institut für Festkörperforschung, Forschungszentrum Jülich GmbH, D-52425 Jülich, Germany
}

(Received 25 July 2003; accepted 16 December 2003)

\begin{abstract}
A systematical computational study of the lattice and rotational dynamics of the methyl halides, which belong to the most simple organic molecules containing $\mathrm{CH}_{3}$ groups, was done. Because of their simplicity there might be a chance to understand and model the dynamics of these systems by combining nonbonded pair interactions and crystallographic information. Based on the experimentally determined crystal structure, which was not relaxed during the calculations, interactions were modeled using the transferable parameters of the universal force fields. The lattice dynamical calculation can reproduce with reasonable accuracy the low-energy regime of the lattice excitations as well as the single-particle rotational potential of the $\mathrm{CH}_{3}$ group of the respective halide. (C) 2004 American Institute of Physics. [DOI: 10.1063/1.1647525]
\end{abstract}

\section{INTRODUCTION}

The theory developed to describe rotational tunneling of molecular crystals is a mean field or single-particle model (SPM) of a quantum-mechanical rotor. ${ }^{1}$ Despite the more recent detection of systems with coupling and multidimensional tunneling, ${ }^{2,3}$ the prominent number of molecular crystals can be well described within the original SPM. ${ }^{4}$ A data analysis going beyond a phenomenological use of this model has to explain the rotational potential on the basis of the crystal structure and fundamental intermolecular interactions. In the standard approach intermolecular interactions are modeled as transferable pair interactions. There is an increasing number of systems analyzed this way. ${ }^{5}$ Their number is mainly limited by the lack of knowledge of a precise low-temperature crystal structure of systems whose tunnel splitting is known from high-resolution neutron spectroscopy.

Typically, it is more difficult to get reliable pair potential parameters for compounds containing atoms heavier than $\mathrm{C}$, $\mathrm{N}$, or $\mathrm{O}$. Whereas potential parameters of pure hydrocarbons are well established, e.g., Refs. 6-8, and allow the description of pure hydrocarbon systems like p-xylene (Ref. 9) or 2-butyne, ${ }^{10}$ it is more difficult to get reliable pair potential parameters from the literature for less common atoms. One successful description of a compound containing $\mathrm{H}, \mathrm{C}$, and $\mathrm{N}, 2,6$-dimethylpyrazine, is given in Ref. 11, another one containing $\mathrm{H}, \mathrm{C}$, and $\mathrm{Br}$ in tribromomesitylene in Ref. 12.

The group of the methyl halides is interesting because they can be used to check hydrogen-halide interatomic pair potentials by consistently describing the lattice dynamics and rotational tunneling of $\mathrm{CH}_{3}$ groups. Since all of the crystal structures are known, a systematic study of this class of mol-

${ }^{a) E l e c t r o n i c ~ m a i l: ~ o k i @ a n s t o . g o v . a u ~}$ ecules is possible. In the present work we describe the lattice dynamics of the molecular crystal by combining the structural information and transferable pair potentials.

\section{A. Crystal structures}

In order to calculate the lattice and the rotational dynamics, a knowledge of the crystallographic structure is required. Whereas the crystal structures of $\mathrm{CH}_{3} \mathrm{Br},{ }^{13} \mathrm{CH}_{3} \mathrm{Cl},{ }^{14} \mathrm{CH}_{3} \mathrm{I}$ (Refs. 15, 16) have been known since the late eighties, the structure of the deuterated fluoride was solved in 1996 by high-resolution powder neutron diffraction. ${ }^{17}$ All of the molecules can be represented as symmetric prolate tops having a small moment of inertia, equal to that of the $\mathrm{CH}_{3}$ group.

If one characterizes the molecular orientation by means of a spin variable then, apart from the chloride, the molecules are arranged as inclined chains in an anti-ferroorientational order. In this description $\mathrm{CH}_{3} \mathrm{Cl}$ represents a ferro-orientational structure. Due to the molecular symmetry there is no intramolecular hindering potential and the methyl rotation is identical to the rotation of the whole molecule around its symmetry axis. Views of the different halides are shown in Fig. 1 and the structural parameters are given in Table I.

\section{B. Experiments}

The lattice and rotational dynamics of the methyl halides were obtained by inelastic neutron scattering experiments. ${ }^{18,19}$ The single-particle excitation energies and derived rotational potentials, which have been compared with the calculations reported in this paper, are given in Table II. Typically, the transition to the first excited librational state is obtained by the temperature-dependent line broadening of the tunneling peak. However, in the case of the fluoride the transition was derived using the temperaturedependent shift of the position of the tunneling peak, assum- 


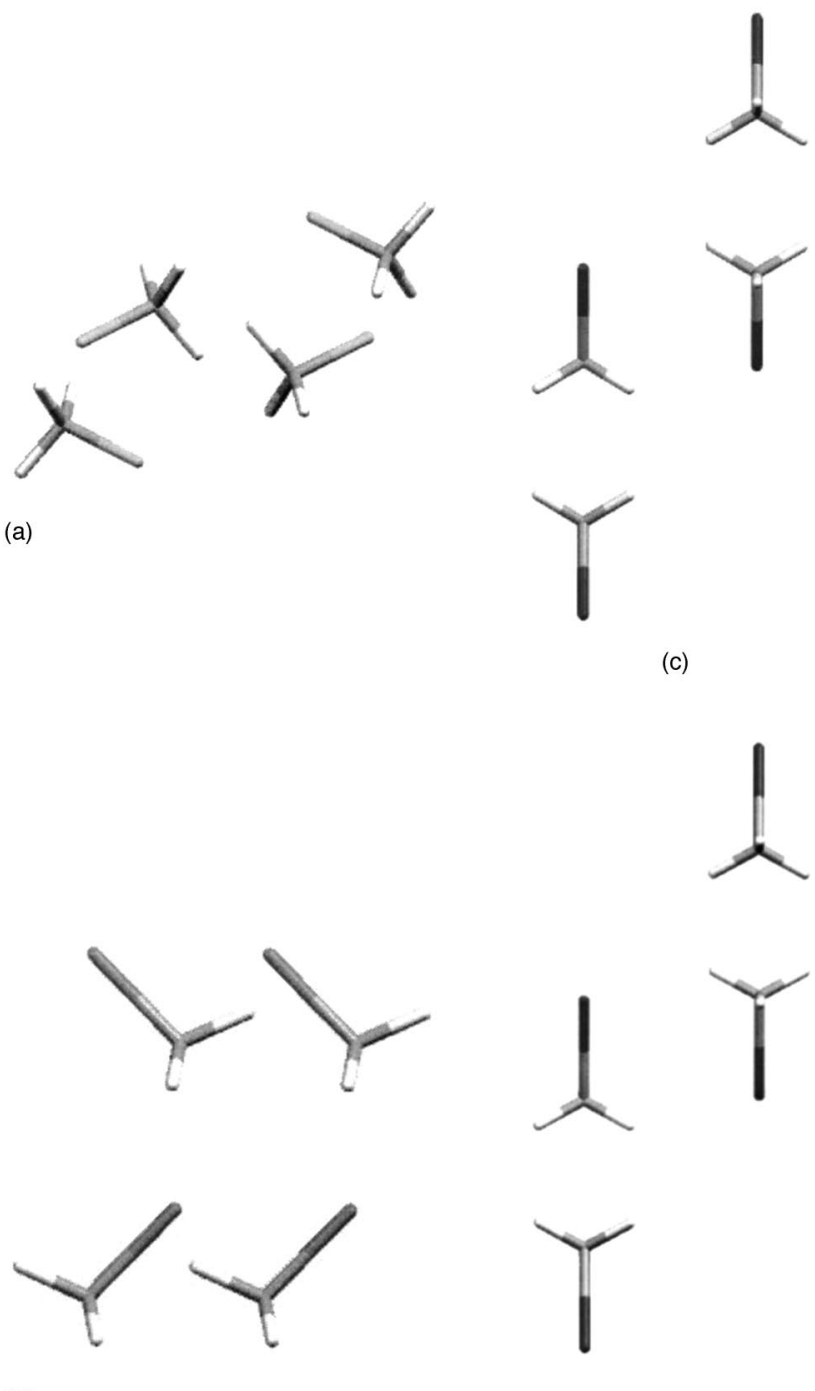

(b)

(d)

FIG. 1. View of the low-temperature unit cells of the methyl halides. Note the ferro-orientational structure of the chloride, contrary to the other halides.

ing a coupling between the rotation of the methyl group and low-energy lattice modes. ${ }^{18}$ The experimental scattering functions $S(Q, \omega)$ are shown in Figs. 2(a)-2(d). One remarkable feature of the fluoride spectrum should be mentioned (see Ref. 19 for a detailed discussion). Normally, the strongest peaks observed in the spectra are caused by the hydrogen atoms because of their large scattering cross section and among these strong peaks the methyl librational modes due to their large displacement amplitudes $u$, see below. These features are clearly visible in Figs. 2(b), 2(c), and 2(d) and are in agreement with the results obtained from the respective high-resolution tunneling experiments. However, in the case of $\mathrm{CH}_{3} \mathrm{~F}$, Fig. 2(a), the librational transition $E_{01}$, derived from the tunneling experiment, is not correlated to the strongest peak in the observed spectrum. The strongest doublets of the other halides can be explained as methyl motions around the molecules symmetry axis $R_{z}$ (=libration) and librations of the whole molecule perpendicular to its symmetry axis $R_{x}$ and $R_{y}$. Since $\mathrm{CH}_{3} \mathrm{~F}$ shows the shortest $\mathrm{C}$-halide bond length, the molecule is less asymmetric. Therefore, the $R_{x, y}$ libration might show larger amplitudes $u$ resulting in an increased intensity in the density of states. This idea is supported by the following argument: There is a systematic increase of the splitting of the $R_{z}$ and the $R_{x, y}$ modes ongoing from the iodide to the fluoride, and the reduced site symmetry of $\mathrm{CH}_{3} \mathrm{~F}$ leads to an enlarged separation of the $R_{x}$ and $R_{y}$ mode visible at 14 and $16 \mathrm{meV}$.

\section{CALCULATIONS}

\section{A. Fundamental theory of lattice dynamics}

The phonon dispersion curves were calculated by the program DYNCAL, which has been successfully applied for similar applications in earlier work..$^{9,10,20}$ Phonon energies and eigenvectors are obtained by solving the set of coupled differential equations $(\alpha, \beta=x, y, z)$

$$
M_{k} \cdot \ddot{u}_{\alpha}(l)=-\sum_{\beta} D_{\alpha \beta}\left(l, l^{\prime}\right) u_{\beta}\left(l^{\prime}\right),
$$

with the force constant matrix $D_{\alpha \beta}\left(l, l^{\prime}\right)$ $=\left[d^{2} V\left(l, l^{\prime}\right)\right] /\left[d u_{\alpha}(l) d u_{\beta}\left(l^{\prime}\right)\right]$ the atomic masses $\mathbf{M}_{k}$, and displacements $\mathrm{u}_{\alpha}$. By using plane waves for $u_{\alpha}(l)$ $=e_{\alpha} / \sqrt{M_{k}} \cdot \exp (i q l-i \omega(q) t)$, the force constant matrix is transformed into the dynamical matrix

$$
\bar{D}_{\alpha \beta}(q)=1 / \sqrt{M_{k} M_{k^{\prime}}} \sum_{l, \beta} D_{\alpha, \beta}(l, 0) \exp (-i q l) .
$$

The eigenvectors and eigenfrequencies are obtained by solving the system $\omega_{0}^{2} \mathbf{e}=\bar{D} \mathbf{e}$ using standard diagonalization procedures.

The force constants characterize the interactomic interactions and are derived in the harmonic approximation as second derivatives of the atom-atom pair potentials $V_{i j}(r)$

TABLE I. Structural properties of the different methyl halides.

\begin{tabular}{lcccc}
\hline \hline & CH3F & CH3Cl & CH3Br & CH3I \\
\hline Cell & $a=6.4373$ & $a=6.4950$ & $a=4.5115$ & $a=4.5368$ \\
parameters & $b=7.5023$ & $b=5.1930$ & $b=6.5040$ & $b=6.8574$ \\
& $c=3.9598$ & $c=7.5230$ & $c=9.3504$ & $c=9.9017$ \\
Space group & $\beta=101.842$ & & & Pnma \\
Z & $P 2 / n$ & $C m c 2$ & Pnma & 4 \\
Geometry & 4 & 4 & 4 & orthorhombic \\
Method & monoclinic & orthorhombic & Neutron diffraction & Neutron diffraction \\
Reference & 17 & X-ray diffraction & 13 & 16 \\
\hline \hline
\end{tabular}


TABLE II. Summary of the excitation energies for the various methyl halides derived by inelastic neutron scattering experiments. $\hbar \omega_{t}=$ tunnel splitting of the ground state; $E_{01}=$ librational energy from the density of states, ${ }^{(1)}$ from the temperature-dependent line broadening $\hbar \omega_{t}(\mathrm{~T}) ; V_{3} / V_{6}$ rotational potential parameters. Values are taken from Ref. 4.

\begin{tabular}{lcccc}
\hline \hline Molecule & $\begin{array}{c}\hbar \omega_{t}^{\text {exp }} \\
\text { in } \mu \mathrm{eV}\end{array}$ & $\begin{array}{c}E_{01}^{\text {exp }} \\
\text { in meV }\end{array}$ & $\begin{array}{c}V_{3}^{\text {exp }} \\
\text { in meV }\end{array}$ & $\begin{array}{c}V_{6}^{\text {exp }} \\
\text { in meV }\end{array}$ \\
\hline $\begin{array}{l}\mathrm{CH}_{3} \mathrm{~F} \\
19\end{array}$ & 23.1 & $10.0^{(1)}$ & 26.6 & 0.0 \\
$\mathrm{CH}_{3} \mathrm{Cl}$ & 0.1 & $18.1_{R_{z}}$ & 79.9 & -5.7 \\
${ }_{18} \mathrm{CH}_{3} \mathrm{Br}$ & 0.9 & $15.8_{R_{z}}$ & 50.7 & 0.6 \\
${ }_{18}$ & 2.44 & $14.57_{R_{z}}$ & 41.0 & 1.9 \\
$\mathrm{CH}_{3} \mathrm{I}$ & & & & \\
\hline
\end{tabular}

with $r=r_{l}-r_{l^{\prime}}$. For our calculations a parametrized BornMeyer ("6-exp") potential was used to describe the nonbonded van der Waals interactions

$$
V_{i j}^{\mathrm{vdW}}(r)=A_{i j} \cdot \exp \left(-B_{i j} \cdot r_{i j}\right)-\frac{C_{i j}}{r_{i j}^{6}}
$$

where the long-range induced dipole interaction is represented by the $r_{i j}^{-6}$ term and the short-range repulsive exponential by the $\exp \left(-B_{i j} \cdot r_{i j}\right)$ term, which in first order is proportional to the overlap of the wave functions squared. The heteroatomic interactions were obtained from the homoatomic parameters by applying the standard combination rules, $A_{i j}=\sqrt{A_{i} \cdot A_{j}}, C_{i j}=\sqrt{C_{i} \cdot C_{j}}$, and $B_{i j}=\frac{1}{2} \cdot\left(B_{i}+B_{j}\right)$, which are commonly known as the conditions of transferability.

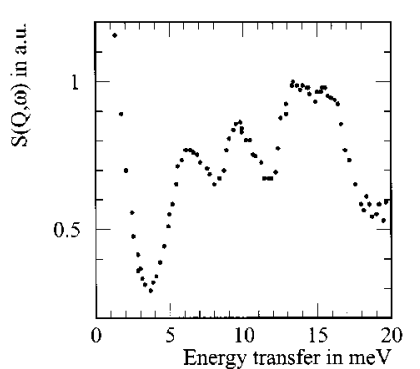

(a)

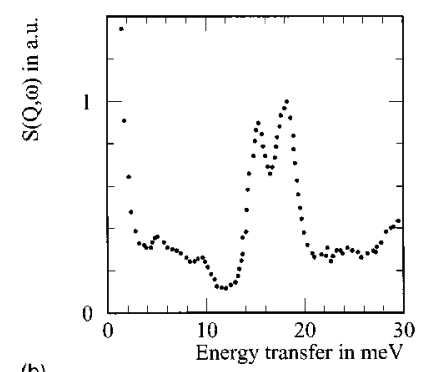

(b)

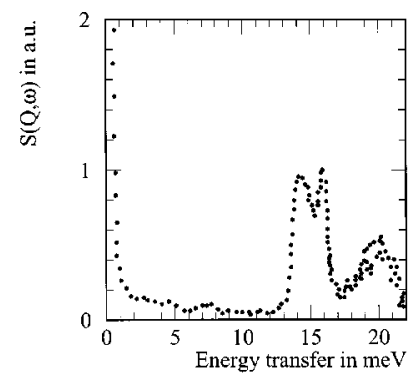

(c)

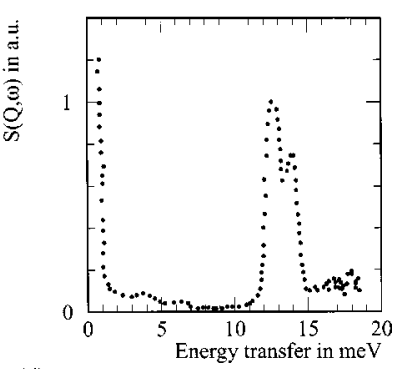

(d)

FIG. 2. Experimental neutron scattering functions $S(Q, \omega)$ for the different methyl halides (Refs. 18, 19).
TABLE III. Parameters of nonbonded pair interactions as given by Ref. 22 .

\begin{tabular}{ccc}
\hline \hline Atom & $x_{i}[\AA]$ & $D_{i}[\mathrm{kcal} / \mathrm{mol}]$ \\
\hline $\mathrm{H}$ & 2.886 & 0.044 \\
$\mathrm{C}$ & 3.851 & 0.105 \\
$\mathrm{~F}$ & 3.364 & 0.050 \\
$\mathrm{Cl}$ & 3.947 & 0.227 \\
$\mathrm{Br}$ & 4.189 & 0.251 \\
$\mathrm{I}$ & 4.500 & 0.339 \\
\hline \hline
\end{tabular}

\section{B. Model of the potential}

\section{1. van der Waals interactions}

The problem of modeling the interactions between the individual atoms of the molecular crystal begins with obtaining the parameters $A, B$, and $C$ for the potential as given in Eq. (1). Pure hydrocarbon system such as p-xylene or toluene have been successfully described by using parameter sets provided by Williams. ${ }^{6}$ Other authors, e.g., Claverie, ${ }^{7}$ Kitaigorodskii, ${ }^{8}$ or Spackman, ${ }^{21}$ provide parameter sets as well, but the parameters given by these authors are only applicable for pure hydrocarbon systems, and either do not include parameters for the halogenides at all or only a subset of the halides under consideration.

Forced to go beyond pure hydrocarbons and access all halogenides simultaneously, we choose to use the parameter set given by Rappé et al. derived within the framework of the Universal Force Fields (UFF). ${ }^{22}$ These authors provide parameters for a variety of elements of the periodic table. The UFF generalizes the Born-Meyer representation of the interaction into an equivalent mathematical form

$$
\begin{aligned}
V_{i j}^{\mathrm{vdW}}(r)= & {\left[D_{i j}\left(\frac{6}{\zeta-6}\right) \exp (\zeta)\right] \exp \left(-\zeta\left(r / x_{i j}\right)\right) } \\
& -\left[D_{i j}\left(\frac{\zeta}{\zeta-6}\right) x_{i j}^{6}\right] /(r)^{6} .
\end{aligned}
$$

The depth of the potential is represented by $D_{i}=C_{i}((\zeta$ $-6) / \zeta) / x_{i}^{6}$ and the repulsive pre-exponential term $A_{i}$ $=D_{i}(6 /(\zeta-6)) \cdot \exp (\zeta)$. The repulsive exponential can be obtained via a scaled distance term, $x_{i}=\zeta / B_{i}$.

The parameters $x_{i}$ and $D_{i}$ fulfill the condition of transferability and allow the calculation of pair interaction parameters. Values chosen for the calculations are taken from Ref. 22 and are listed in Table III. The parameter $\zeta$ can be chosen to have a value of $12,13.772$, or to be individually fitted to a discrete set of crystal structures for each element. For our calculation we choose $\zeta=12$, which results in the "6-exp" potential; see Eq. (1). A similar approach was chosen by Nicolai et al. for the description of 2,6-dimethylpyrazine (Ref. 11) and Errington et al. for simulating the properties of water-methane and water-ethane mixtures. ${ }^{23}$

\section{Electrostatic interactions}

In addition to the van der Waals contribution of the potential, the electrostatic interaction has been taken into account by an additional term 
TABLE IV. Charges $n_{i}$ obtained using $a b$ initio quantum-mechanical calculations. The charges are given in units of the elementary charge $q_{0}$.

\begin{tabular}{lccc}
\hline \hline $\mathrm{CH}_{3} \mathrm{~F}$ & $\mathrm{CH}_{3} \mathrm{Cl}$ & $\mathrm{CH}_{3} \mathrm{Br}$ & $\mathrm{CH}_{3} \mathrm{I}$ \\
\hline $\mathrm{H}: 0.165$ & $\mathrm{H}: 0.228$ & $\mathrm{H}: 0.092$ & $\mathrm{H}: 0.268$ \\
$\mathrm{C}:-0.162$ & $\mathrm{C}:-0.578$ & $\mathrm{C}:-0.266$ & $\mathrm{C}:-0.834$ \\
$\mathrm{~F}:-0.332$ & $\mathrm{Cl}:-0.105$ & $\mathrm{Br}:-0.010$ & I: 0.032 \\
\hline \hline
\end{tabular}

$$
V_{i j}^{\mathrm{el}}(r)=\frac{n_{i} \cdot n_{j} \cdot q_{0}^{2}}{r_{i j}} .
$$

Here, $n_{i}$ and $n_{j}$ are the charges in units of the elementary charge $q_{0}$. The charge distribution within the individual halide is modeled by point charges located on atomic sites. The geometry of the respective molecule is taken from the crystal structure information, Sec. $1 \mathrm{~A}$, and was not modified during the calculations. ${ }^{24}$ For each molecule a density functional calculation, as implemented in the GAUSSIAN 98 program package, ${ }^{25}$ was performed using a $6-31 \mathrm{G}^{*}$ basis set to calculate point charges for each atom of the respective halide. The charges obtained are given in Table IV.

Taking the van der Waals and electrostatic interactions into account, the potential energy of the individual crystal may be written as $V_{i j}=\frac{1}{2} \sum_{i j}\left(V_{i j}^{\mathrm{vdW}}+V_{i j}^{\mathrm{el}}\right)$. The nonbonded interactions have been calculated using the Ewald summation method; see, e.g., Ref. 26. For computational reasons the potential was described by a power series, $V_{i j}$ $=\sum_{l=1}^{k}\left[\alpha_{i j}(l)\right] /\left[r_{i j}^{n(l)}\right]$, because the summation converges much faster than by calculating the exponential function. ${ }^{27}$ We used 6 terms in the expansion with $n(l)=4,6,8,10,12$, 14. The parameters $\alpha_{i j}(l)$ were adjusted to describe the interactions within the range of $2<r<5 \AA$; see Fig. 3 .

\section{Model of the molecule}

\section{Semirigid molecule}

Besides the pair potentials DYNCAL requires the crystal structure in order to obtain the interatomic distances. When modeling molecular crystals it is often not necessary to deal with all $3 N$ independent Cartesian coordinates of the individual atoms, and it is usually more economical to adopt the

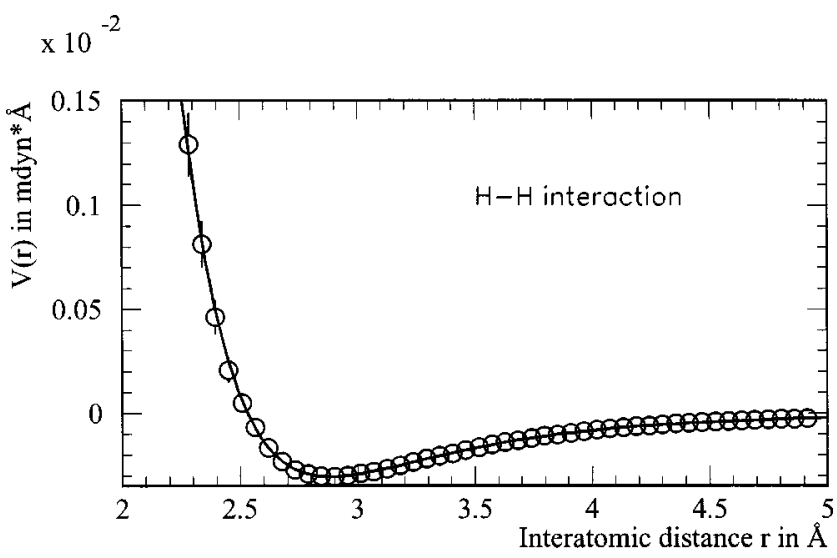

FIG. 3. UFF pair interaction potential for a hydrogen-hydrogen interaction. $\bigcirc$ are calculated values; the line represents a fit with six power functions; see the text. number of degrees of freedom to the problem. The most extreme and widely used form of reducing the degrees of freedom is the assumption of a completely rigid molecule. Of course, many molecules cannot be considered as completely rigid but it may be possible to identify rigid subgroups within the molecule. Rigid and semirigid molecules may be specified by a number of geometrical constraints, e.g., fixed bond lengths, fixed torsional angles, or the confinement of atoms to one plane. In the present case we model the molecule to have no internal degree of freedom. Because of this restriction intramolecular modes such as stretching or bending modes have been excluded from the calculations.

\section{The neutron scattering function and the density of states}

The scattering function $S(Q, \omega)$ in the meV range is typically observed in an inelastic neutron scattering experiment and contains the weighted phonon density of states (DOS) which is calculated by the lattice dynamics program as a weighted average of all excitations

$$
\begin{gathered}
S(Q, \omega) \sim \frac{1}{2} \sum_{i} \sigma_{i} \sum_{l} \sum Q_{j}^{2}\left\langle u_{i j l}^{2}\right\rangle \cdot e^{-Q^{2} u_{i j l}^{2}} \\
\cdot \delta\left(\omega-\omega_{j}\left(q_{l}\right)\right) .
\end{gathered}
$$

The summation runs over the displacements $u_{i j l}$ of all atoms $i$ in the unit cell according to eigenmode $j$ on a threedimensional grid of momentum transfers $q_{l}$ covering the Brillouin zone homogeneously. A Debye-Waller factor $\exp \left(-Q^{2} u_{i j l}^{2}\right)$ is included. The calculated scattering function corresponds to the neutron scattering function according to the model of Zemach and Glauber in the regime of phonons and librons. ${ }^{28}$ The summation $l$ over the Brillouin zone is required due to phonon dispersion. Methyl motions usually show large mean-square displacements $\left\langle u_{i j l}^{2}\right\rangle$. Furthermore, they involve the proton with its dominant scattering cross section $\sigma_{i}$, which often results in the strongest peaks in the spectrum.

\section{E. Methyl group rotation}

Besides the classical lattice dynamics, the rotational tunneling of the methyl group must be described by the chosen transferable pair potential, too. For this purpose a mean-field rotational potential is calculated using the same pair interactions as for the lattice dynamics. Therefore, a methyl group is rotated about the bond axis while all other atoms are fixed at their equilibrium positions. Adding up, for each $\mathrm{CH}_{3}$ orientation, the pair interactions of the three methyl protons with all the other lattice atoms yields the potential energy of this configuration. If one would rotate the $\mathrm{CH}_{3}$ group, as determined crystallographically with all the positional error bars, as a rigid unit the proton sites would only coincide after a rotation of $2 \pi$. Thus, when calculating $V(\varphi)$ we force the methyl protons to move on the common circle of circumference between equilibrium positions of the two neighboring methyl protons. This procedure makes the protons coincide exactly after $2 \pi / 3$. The angle-dependent part of the potential energy can be identified as the mean-field methyl rotational 


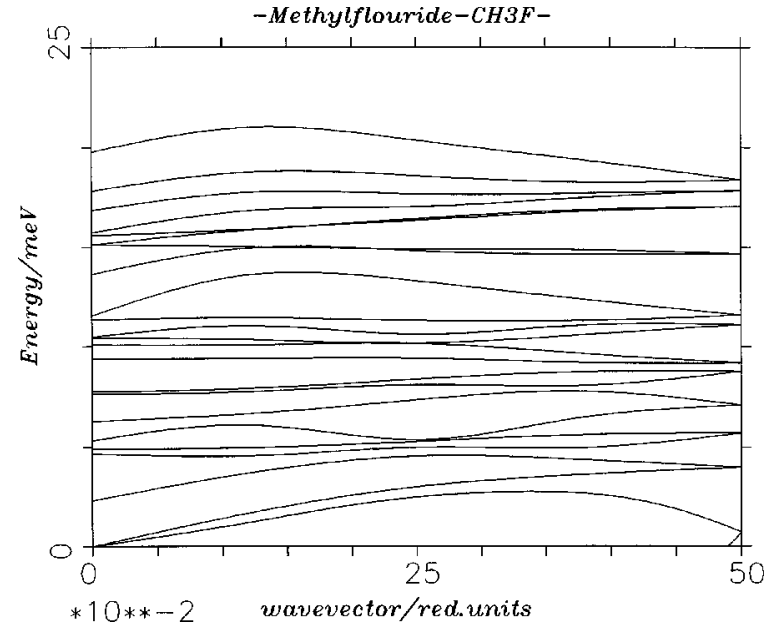

FIG. 4. Dispersion curves along the [100] direction for $\mathrm{CH}_{3} \mathrm{~F}$. The absence of one acoustic branch at the $\Gamma$ point, which appears close to the [100] boundary, indicates that the crystal is not stable under the given potential.

potential $V(\varphi)$. For further analysis $V(\varphi)$ is described by a Fourier series. Due to the limited experimental information available, the series is typically truncated to a second-order series

$$
V(\varphi)=V_{0}+\sum_{n=1}^{2} \frac{V_{3 n}}{2}\left(1-\cos \left(3 n \varphi+\alpha_{3 n}\right)\right)
$$

In the single-particle approach the rotational excitations are solutions of the time-independent Schrödinger equation

$$
\begin{aligned}
& H\left|\Upsilon_{i}\right\rangle=E_{i}\left|\Upsilon_{i}\right\rangle, \\
& -\left[\frac{\hbar^{2}}{2 I} \frac{d^{2}}{d \varphi^{2}}-V(\varphi)\right]\left|\Upsilon_{i}\right\rangle=E_{i}\left|\Upsilon_{i}\right\rangle,
\end{aligned}
$$

with the molecular moment of inertia $I$ and the rotational constant $B_{\text {rot }}=\hbar^{2} / 2 I$ of the methyl group. The spatial wave functions are expanded into free rotor functions $|m\rangle$ $\propto \exp (\operatorname{im} \varphi)$ according to $Y_{i}=\Sigma_{m} \alpha_{m}|m\rangle$, with the eigenvector $\boldsymbol{\alpha}$. The eigenvalues are calculated by diagonalizing the Hamilton matrix $H$ with matrix elements

$$
H_{m n}=\langle m|H| n\rangle,
$$

using standard procedures.

\section{RESULTS}

\section{A. Density of states-S $Q, \omega)$ and $\mathrm{CH}_{3}$ rotation}

Before discussing the dispersion curves obtained from DYNCAL, we would like to emphasize that we used the information provided by the low-temperature crystal structure of the respective halide, Sec. I A, as an experimental fact measured with high precision. Therefore, we did not "optimize" the experimentally determined crystal structure and did not perform a geometry optimization followed by a calculation of the crystal properties. Typical phonon dispersion curves of the individual molecule along the [100] direction are shown in Figs. 5(a)-5(d).

The powder spectra were calculated according to Eq. (4) and are shown with the experimental spectra in Figs. 6(a)-

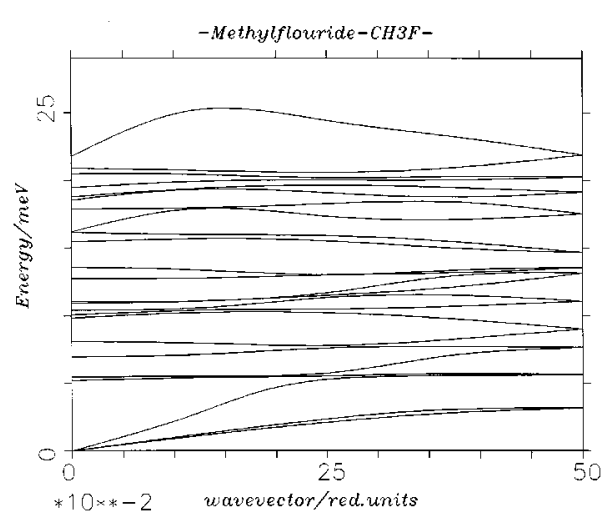

(a)

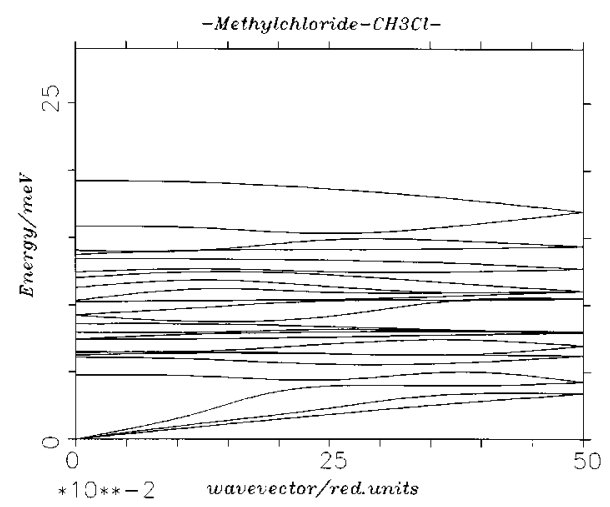

(b)

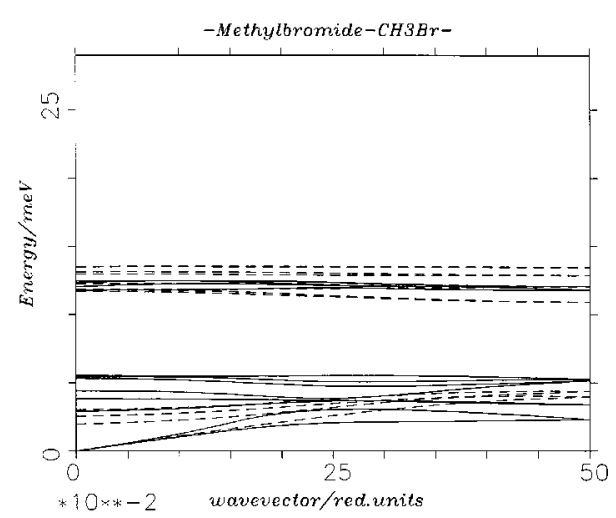

(c)

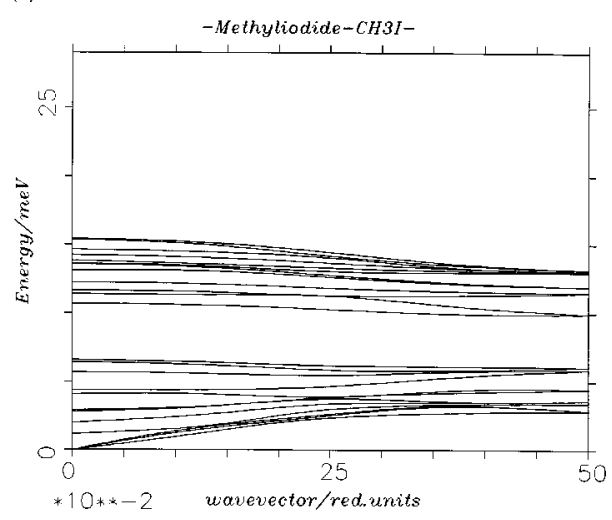

(d)
FIG. 5. Phonon dispersion curves of the individual methyl halides along the [100]-direction. (a) Methylfluoride; (b) methylchloride; (c) methylbromide; (d) methyliodide. 


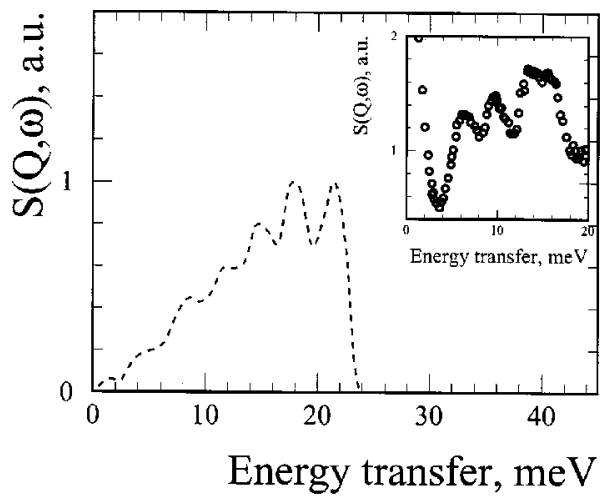

(a)

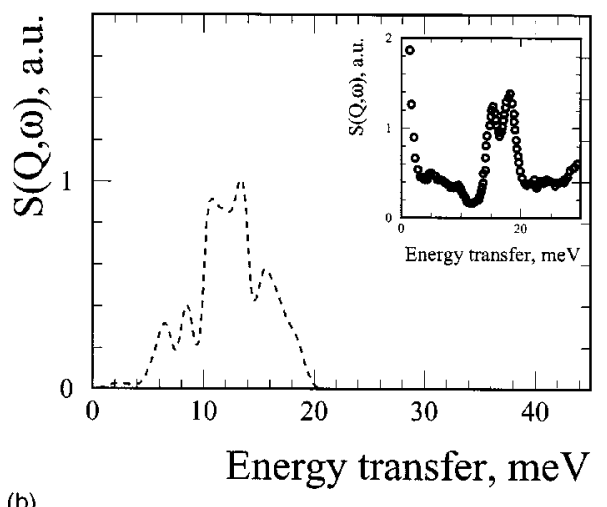

(b)

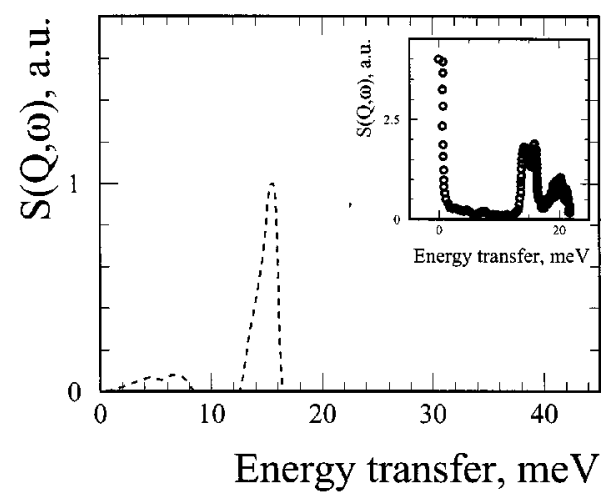

(c)

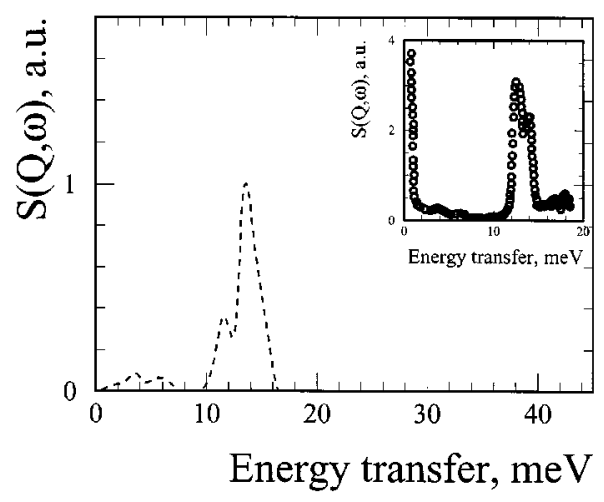

(d)
FIG. 6. Calculated scattering function $S(Q, \omega)$ of the individual methyl halides. The experimental librational transition $R_{z}$ as well as the transition calculated within the SPM is marked. The inserts show the experimental spectra. (a) Methylfluoride; (b) methylchloride; (c) methylbromide; (d) methyliodide. 6(d). The derived rotational potentials, obtained within the SPM, which determine the tunneling frequencies $\hbar \omega_{t}^{\mathrm{spm}}$ and the librational transitions $E_{01}^{\mathrm{spm}}$, as well as the activation energies $E_{a}^{\mathrm{spm}}$, are given in Table $\mathrm{V}$. The properties of the resulting spectra and the corresponding rotational potentials are discussed in the following sections.

\section{1. $\mathrm{CH}_{3} \mathrm{~F}$}

The first step when calculating the dynamics of the respective molecular system is to reproduce the static or equilibrium properties of the individual system. A necessary condition for the correctness of the lattice dynamics calculation is that it should not deliver any negative phonon frequencies. A first calculation for the methylfluoride yielded negative eigenfrequencies of about to $-2.5 \mathrm{meV}$ at the $\Gamma$ point, indicating that the crystal was not in equilibrium under the given potential; see Fig. 4.

One possibility of stabilizing the crystal is to modify the parameters of the potential while keeping the crystallographic structure fixed. The potential parameters for $\mathrm{H}$ and $\mathrm{C}$ should not be modified to obtain their transferability and make sure that they can be applied for the other halides as well. Therefore, the potential parameters for the fluor are accessible to modifications only. One way of modifying fluor interactions is by applying a small variation in the interatomic distance $\delta r, r \Rightarrow r+\delta r$, so that the repulsive exponential term in Eq. (1) becomes

$$
A \cdot \exp (-B \cdot r) \Rightarrow A \cdot \exp (-B \cdot(r+\delta r)) .
$$

The variation was applied to the interaction $\mathrm{H}-\mathrm{F}, \mathrm{C}-\mathrm{F}$, and $\mathrm{F}-\mathrm{F}$ only. The dynamical calculation yielded a stable system (=no negative eigenfrequencies) for $\delta r=-0.1$. One possible explanation for modifying the potential may be the presence of $\mathrm{H} \cdots \mathrm{F}$ bonds, which have not been explicitly modeled.

By inspection of the eigenmodes, after the stable crystal system was obtained, the mode at $11 \mathrm{meV}$ was identified as librational excitation within the SPM correlated to $R_{z}$. Only a small amount of dispersion occurs for this mode, indicating only a small mode coupling $\omega(q)$ $\propto \sqrt{\left[d^{2} V\left(l, l^{\prime}\right)\right] /\left[d u_{\alpha}(l) d u_{\beta}\left(l^{\prime}\right)\right]}$. The higher peaks are due to $R_{x, y}$ motions of the hydrogen atoms. The calculation thus endorses the assumption of Prager et al. ${ }^{19}$ that the $R_{z}$ libration does not correspond to the strongest peak in the experi-

TABLE V. Summary of the calculated parameters for the various methyl halides using the SPM model.

\begin{tabular}{lcccccrrr}
\hline \hline Molecule & $\begin{array}{c}\hbar \omega_{t}^{\text {spm }} \\
\text { in } \mu \mathrm{eV}\end{array}$ & $\begin{array}{c}E_{0}^{\text {spm }} \\
\text { in meV }\end{array}$ & $\begin{array}{c}E_{01}^{\text {spm }} \\
\text { in meV }\end{array}$ & $\begin{array}{c}E_{a}^{\text {spm }} \\
\text { in meV }\end{array}$ & $\begin{array}{c}V_{3}^{\text {spm }} \\
\text { in meV }\end{array}$ & $\begin{array}{c}V_{6}^{\text {spm }} \\
\text { in meV }\end{array}$ & $\begin{array}{c}\alpha_{3}^{\text {spm }} \\
\text { in deg }\end{array}$ & $\begin{array}{c}\alpha_{6}^{\text {spm }} \\
\text { in deg }\end{array}$ \\
\hline $\mathrm{CH}_{3} \mathrm{~F}$ & 24.4 & 8.6 & 11.3 & 16.4 & 18.2 & 10.9 & 11.0 & -23.3 \\
$\mathrm{CH}_{3} \mathrm{Cl}$ & 2.2 & 5.4 & 12.1 & 45.7 & 51.1 & -10.1 & 0.0 & 0.0 \\
$\mathrm{CH}_{3} \mathrm{Br}$ & 0.4 & 7.8 & 15.7 & 57.1 & 64.8 & -6.4 & 0.0 & 0.0 \\
$\mathrm{CH}_{3} \mathrm{I}$ & 1.7 & 5.8 & 12.6 & 47.1 & 52.9 & -9.5 & 0.0 & 0.0 \\
\hline \hline
\end{tabular}




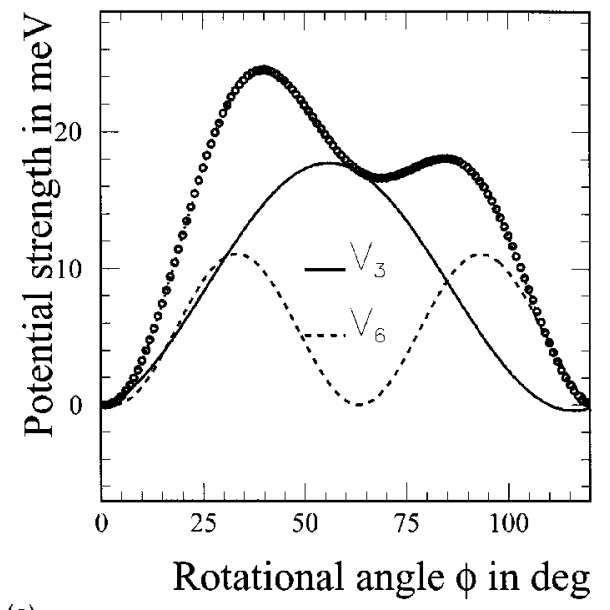

(a)

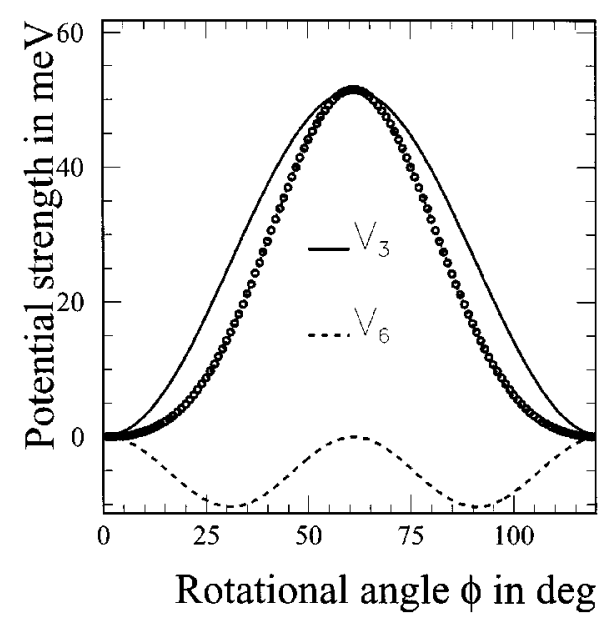

(b)

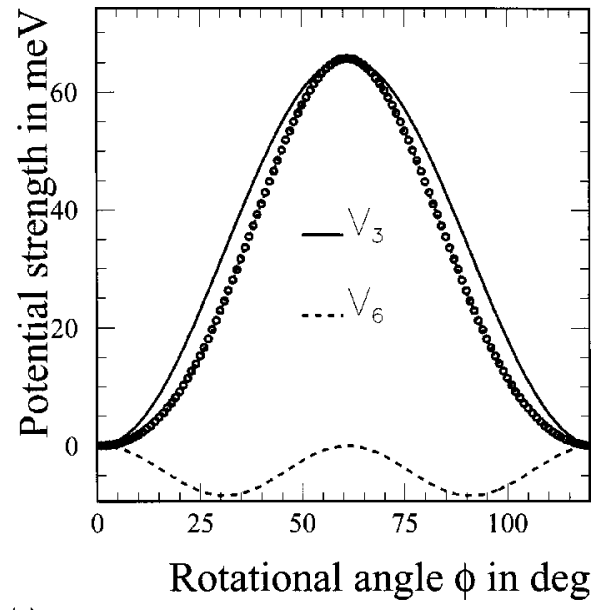

(c)

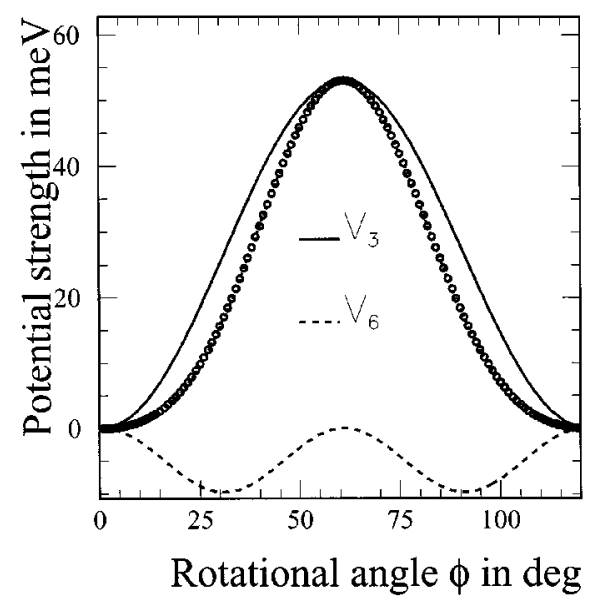

(d)
FIG. 7. Calculated rotational potentials $\mathrm{V}(\varphi)$. (a) Methylfluoride; (b) methylchloride; (c) methylbromide; (d) methyliodide. $\bigcirc$ represent the calculated $V(\varphi)$; the lines represent the fit according to Eq. (5). mentally observed spectrum, as noted earlier in Sec. I B. The prominent features of the scattering function are reproduced as follows: Four dominant peaks are visible at $8 \mathrm{meV}, 11$ $\mathrm{meV}$ (=libration), $16 \mathrm{meV}$, and a doublet at 18 and $22 \mathrm{meV}$, Fig. 6(a). The minima of the appendant rotational potential do not differ from the crystallographically determined equilibrium orientation, Fig. 7(a). The $V_{3}$ term of the potential representing the $C_{3}$ symmetry of the rotator shows a phase shift, which is compensated by the weaker $V_{6}$ term representing the environment seen by the rotator. The calculated splitting due to tunneling of $24.4 \mu \mathrm{eV}$ is very close to the experimental value of $23.1 \mu \mathrm{eV}$, especially by taking into account the exponential dependence of the potential. The relative phases of the rotational potential are accessible to calculations only. In the experiment ${ }^{19}$ only two observables were obtained and the experimentally determined parameters of the potential assume $\alpha_{3}=\alpha_{6}=0$. The good agreement between the SPM model, the lattice dynamics, and the experiment justifies the introduced modification of the fluor interactions, although this procedure is, of course, not a refinement of the UFF parameters but became necessary to obtain a stable crystal.

\section{2. $\mathrm{CH}_{3} \mathrm{Cl}$}

The dispersion curves obtained for the methyl chloride represent a stable system showing no negative energies, Fig. 5(b). There is little dispersion in the regime of the optical phonon branches. The calculated scattering function $S(Q, \omega)$ of the chloride shows peaks at around $12 \mathrm{meV}$ with doublet characteristics (11.7 and $12.9 \mathrm{meV})$. Some further intensity is visible at about $16 \mathrm{meV}$. Based on the characteristics of the respective eigenvectors, the peak at $12.9 \mathrm{meV}$ is identified as being the $R_{z}$ libration. The $R_{x, y}$ librations cause the calculated intensity at about $\sim 11.7 \mathrm{meV}$. Although the characteristics of the calculated $S(Q, \omega)$ reproduce the main characteristics of the experimental one, there is a clear deviation between the calculated and experimental librational energies. The minimum of the calculated rotational potential coincides well with the crystallographic equilibrium orientation of the methyl group. The $C_{3}$ symmetry is represented by a dominant $V_{3}$ part of the potential without a phase shift, Fig. 7(b); neither does the smaller $V_{6}$ contribution of the potential. The calculated tunneling splitting is $2.6 \mu \mathrm{eV}$, being higher than the experimental value of $0.1 \mu \mathrm{eV}$.

\section{3. $\mathrm{CH}_{3} \mathrm{Br}$}

All of the calculated modes for the methylbromide show positive energies. As with the chloride the dispersion curves again indicate only a small coupling in the optical regime. Therefore, the first librational transition $E_{01}$ is assigned to the single-particle librational transition. The calculated scat- 
TABLE VI. Calculated librational transitions and activation energies using the harmonic oscillator approximation. Differences between $E_{0}^{\mathrm{ho}} / E_{0}^{\mathrm{spm}}, E_{01}^{\mathrm{ho}} / E_{01}^{\mathrm{spm}}\left(\right.$ Table V), and $E_{01}^{\mathrm{ho}} / E_{01}^{\mathrm{spm}}$ are given as $\Delta_{E_{0}}, \Delta_{E_{01}}$, and $\Delta_{E_{a}}$ in $\%$.

\begin{tabular}{lccccrr}
\hline \hline Molecule & $\begin{array}{c}E_{0}^{\text {ho }} \\
\text { in meV }\end{array}$ & $\begin{array}{c}E_{01}^{\text {ho }} \\
\text { in meV }\end{array}$ & $\begin{array}{c}E_{a}^{\text {ho }} \\
\text { in meV }\end{array}$ & $\begin{array}{c}\Delta_{E_{0}} \\
\text { in \% }\end{array}$ & $\begin{array}{c}\Delta_{E_{01}} \\
\text { in \% }\end{array}$ & $\begin{array}{c}\Delta_{E_{a}} \\
\text { in \% }\end{array}$ \\
\hline $\mathrm{CH}_{3} \mathrm{~F}$ & 5.1 & 10.3 & 14.7 & 40 & 9 & 10 \\
$\mathrm{CH}_{3} \mathrm{Cl}$ & 4.1 & 8.2 & 43.0 & 25 & 33 & 6 \\
$\mathrm{CH}_{3} \mathrm{Br}$ & 7.6 & 15.2 & 49.6 & 3 & 3 & 13 \\
$\mathrm{CH}_{3} \mathrm{I}$ & 4.7 & 9.4 & 43.5 & 19 & 25 & 8 \\
\hline \hline
\end{tabular}

tering function of the bromide shows the prominent features of the measured spectrum, Fig. 6(c): At first, there is intensity below $8 \mathrm{meV}$ followed by a gap around $10 \mathrm{meV}$. Peaks with doublet characteristic occur at 14 and $16 \mathrm{meV}$. The $R_{z}$ libration and $R_{x, y}$ motion of the molecules are resolved as separate peaks and, by inspecting the eigenmodes, the peak at $16 \mathrm{meV}$ is assigned to $R_{z}$, the $14 \mathrm{meV}$ peak to $R_{x, y}$. The value for the transition to the first librational transition, calculated in the SPM, is $E_{01}=15.7 \mathrm{meV}$. Regarding the rotational potential, the $\mathrm{C}_{6}$ contribution is low compared to the dominant $\mathrm{C}_{3}$ part representing the symmetry of the methyl group. The equilibrium orientation is also well reproduced and the respective potential contributions do not show a relative phase shift, Fig. 7(c). The SPM tunneling splitting is calculated to be $0.4 \mu \mathrm{eV}$.

\section{4. $\mathrm{CH}_{3} \mathrm{I}$}

Only a small amount of mode coupling is visible in the dispersion curves representing a stable system with no negative eigenfrequencies; see Fig. 5(d). The calculated scattering function of the iodide, Fig. 6(d), shows peaks with doublet characteristics at about 12 and $14 \mathrm{meV}$. The individual $R_{x, y}$ and $R_{z}$ transitions are separated and the $14 \mathrm{meV}$ peak is due to the $R_{z}$ libration. The calculated value for $E_{01}^{\mathrm{spm}}$ is $12.6 \mathrm{meV}$ and can be interpreted as being an average librational transitions even with respect to the small coupling in the regime of the optical phonon branches.

The rotational potential; Fig. 7(d), reveals that the equilibrium structure is well reproduced by the shape of the potential. The calculated splitting due to tunneling of $1.7 \mu \mathrm{eV}$ is in good agreement with the experimental value of $2.4 \mu \mathrm{eV}$.

\section{Effect of anharmonicity}

In this section we give an estimate of the effect of anharmonicity on the excitations, which is relevant for the librations because of the large mean-square displacement $u$ of the hydrogen atoms. To do this we compare the values for the transition to the first excited state $E_{01}$, obtained by solving Eqs. (6) and (7), with the results obtained by assuming a simple harmonic oscillator whose equidistant energy levels are given by

$$
E_{\nu}^{\mathrm{ho}}=\left(\nu+\frac{1}{2}\right) \cdot \sqrt{2 \cdot B \cdot \frac{d^{2}}{d \varphi^{2}} V(\varphi)} .
$$

The potential $V(\varphi)$ is given by a Fourier series according to Eq. 5. We apply the relation $\cos (l \varphi+\alpha) \approx \cos (\alpha)-l \varphi \cdot \sin (\alpha)$ $-\left[(l \varphi)^{2} / 2 !\right] \cos (\alpha)$ and require $(d / d \varphi) V(\varphi)=0$. This leads to

$$
\begin{aligned}
& E_{\nu}^{\mathrm{ho}}=\frac{3}{2}(2 \nu+1) \cdot \sqrt{B \cdot\left(V_{3} \cos \alpha_{3}+4 \cdot V_{6} \cos \alpha_{6}\right)}, \\
& E_{01}^{\mathrm{ho}}=E_{1}^{\mathrm{ho}}-E_{0}^{\mathrm{ho}}=2 \cdot E_{0}^{\mathrm{ho}} .
\end{aligned}
$$

The values $E_{01}$ and $E_{a}$ using the harmonic oscillator approximation were calculated for each individual compound and are given in Table V. Since Eq. (8) is valid for $V_{3} \cos \alpha_{3}$ $\geqslant 4 \cdot V_{6} \cos \alpha_{6}$, only the threefold term in case of the methylfluoride has been taken into account.

The smallest differences between the analytical SPM values and the harmonic oscillator approximation with regards to the ground state, transition to the first librational state, and the activation energy occurs for the methylbromide. The close agreement is also clear if the calculated and experimental values for the tunneling transition and scattering functions $S(Q, \omega)$ are compared, as can be seen in Fig. 6(c).

Another representation of the SPM potential is given by its strength defined as $V S=\left|V_{3}\right|+\left|V_{6}\right|$ and its deviation from a pure threefold potential $\delta=V_{3} / V S$. Again, using the values for the bromide the strength of the potential is $V S$ $=71.2 \mathrm{meV}$ and $\delta=0.91$. Of course, the bigger $V S$ and $\delta$ the better justified is the lattice dynamics calculation using the harmonic approximation. Looking at Table VI one would expect a sequence "bromide-iodide-chloride." Even the calculations for the fluoride with its "soft" potential and the strong sixfold component of the rotational potential show a reasonable agreement with the experiment.

\section{DISCUSSION}

A systematic computational study of the motion of all four methyl-halides within the model of semirigid molecules has been carried out. It was shown that it is possible to describe simultaneously the dynamics of the crystal lattice and the anharmonic motion of the $\mathrm{CH}_{3}$ groups, including the rotational tunneling by the same set of intermolecular potentials, based on the parameters of the Universal Force Fields with reasonable accuracy. The important point is that the structural and position information obtained by highresolution powder diffraction has not been modified to reproduce the experimentally obtained scattering functions and potential parameters, which describe the low-energy dynamics of the individual molecular crystal. While the agreement between the calculations and experiments is reasonable for the methylchloride, methylbromide, and methyliodide, agreement and a stable system for the methylfluoride was obtained only by varying the strength of the short-range exponential term in the potential. This variation may be a hint for the presence of additional contributions to the potential 
such as $\mathrm{H} \cdots \mathrm{F}$ bonds, which have not been explicitly modeled. The calculated librations in case of the methylchloride and the methyliodide are indicative that the contribution of the respective halide to the potential may have to be refined to make the potential stronger, which would increase the values obtained for the librational transitions and decrease the tunnel splittings. The best agreement between the experiment and the calculation was obtained for the methylbromide, indicating that the parameters given by the UFF are suitable to model the $\mathrm{Br}$ interactions.

For all compounds, the application of the harmonic approximation gives reasonable agreement with the experimental data and with the "exact" solutions obtained by diagonalizing the Hamilton matrix within the SPM. The calculated scattering functions $S(Q, \omega)$ exhibit the most prominent features which are visible in the experimental spectra. The dispersion curves indicate in all cases stable systems without significant dispersion of the methyl librations, and therefore only weak coupling between the librations and other lattice modes. The dynamics modeled in the harmonic approximation should be a good approximation for the chloride, bromide, and the iodide with respect to the individual potential strength $\delta$.

The calculations also predict values for the activation energies of the respective halide. Since, to our knowledge, no activation energies have yet been determined, we intend to start an experiment to obtain these activation energies by means of quasielastic neutron scattering. The results will then be used to check the values calculated. Finally, the parameters provided by the Universal Force Fields offer the possibility to characterize systems far beyond pure hydrocarbons if it is possible to combine the structural information and potential parameters within the single-particle model.

\section{ACKNOWLEDGMENT}

One of us (O.K.) would like to thank Dr. M. E. Hagen for his interest and all the discussions related to this paper.
${ }^{1}$ W. Press, Single Particle Rotations in Molecular Crystals, Springer Tracts in Modern Physics (Springer, Berlin, 1981), Vol. 81.

${ }^{2}$ P. Schiebel, A. Hoser, W. Prandl, G. Heeger, W. Paulus, and J. Schweiss, J. Phys.: Condens. Matter 6, 10989 (1994).

${ }^{3}$ V. A. Benderskii, E. V. Vetoshkin, S. Yu. Grebeshchikow, L. von Laue, and H. P. Tromsdorff, Chem. Phys. 219, 143 (1997).

${ }^{4}$ M. Prager and A. Heidemann, Chem. Rev. 97, 2933 (1997).

${ }^{5}$ M. R. Johnson, M. Neumann, B. Nicolai, P. Smith, and G. J. Kearley, Chem. Phys. 215, 343 (1997).

${ }^{6}$ D. E. Williams, J. Chem. Phys. 47, 4680 (1967).

${ }^{7}$ J. Caillet and P. Claverie, Acta Crystallogr. 18, 585 (1965).

${ }^{8}$ A. I. Kitaigorodskii, K. V. Mirskaya, and V. V. Nauchitel, Kristallografiya 9, 900 (1969).

${ }^{9}$ M. Prager, W. I. F. David, and R. M. Ibberson, J. Chem. Phys. 95, 2473 (1991).

${ }^{10}$ O. Kirstein, M. Prager, M. R. Johnson, and S. F. Parker, J. Chem. Phys. 117, 1313 (2002).

${ }^{11}$ B. Nicolaï, E. Kaiser, F. Fillaux, G. J. Kearley, A. Cousson, and W. Paulus, Chem. Phys. 226, 1 (1998).

${ }^{12}$ J. Meinnel, M. Mani, A. Cousson, F. Boudjada, W. Paulus, and M. Johnson, Chem. Phys. 261, 165 (2000).

${ }^{13}$ P. N. Gerlach, B. H. Torrie, and B. M. Powell, Mol. Phys. 57, 919 (1986).

${ }^{14}$ R. D. Burbank, J. Am. Chem. Soc. 75, 1211 (1953).

${ }^{15}$ M. Kawaguchi, M. Hijikigawa, Y. Hayafuji, M. Ikeda, R. Fukushima, and Y. Tomie, Bull. Chem. Soc. Jpn. 46, 53 (1973).

${ }^{16}$ R. M. Ibberson and M. Prager (unpublished).

${ }^{17}$ R. M. Ibberson and M. Prager, Acta Crystallogr., Sect. B: Struct. Sci. B52, 892 (1996).

${ }^{18}$ M. Prager, J. Stanislawski, and W. Häusler, J. Chem. Phys. 86, 2563 (1987).

${ }^{19}$ M. Prager, J. Chem. Phys. 89, 1181 (1988).

${ }^{20}$ J. Kalus, M. Monkenbusch, I. Natkaniec, M. Prager, J. Wolfrum, and F. Wörlen, Mol. Cryst. Liq. Cryst. Sci. Technol., Sect. A 268, 1 (1995).

${ }^{21}$ M. A. Spackman, J. Chem. Phys. 85, 6579 (1986).

${ }^{22}$ A. K. Rappé, C. J. Casewitt, K. S. Colwell, W. A. Goddard III, and W. M. Skiff, J. Am. Chem. Soc. 114, 10024 (1992).

${ }^{23}$ J. R. Errington, G. C. Boulougouris, I. G. Economou, A. Z. Panagiotopoulos, and D. N. Theodorou, J. Phys. Chem. B 102, 8865 (1998).

${ }^{24}$ G. Rauhaut and P. Pulay, J. Phys. Chem. 99, 3093 (1995).

${ }^{25}$ M. J. Frisch, G. W. Trucks, H. B. Schlegel, et al., GAUSSIAN 98, REVISION A 7, Gaussian, Inc., Pittsburgh, PA, 1998.

${ }^{26}$ E. W. Kellermann, Philos. Trans. R. Soc. London, Ser. A 238, 513 (1940).

${ }^{27}$ M. Monkenbusch, Comput. Phys. Commun. 67, 343 (1991).

${ }^{28}$ A. C. Zemach and R. J. Glauber, Phys. Rev. 101, 118 (1956). 